\title{
Concerning Spin as Mind-pixel: How Mind Interacts with the Brain through Electric Spin Effects
}

\begin{abstract}
Huping $\mathrm{Hu}$ and Maoxin $\mathrm{Wu}$
Abstract: Electric spin effects are effects of electric fields on the dynamics/motions of nuclear/electron spins and related phenomena. Since classical brain activities are largely electric, we explore here a model of mind-brain interaction within the framework of spin-mediated consciousness theory in which these effects in the varying high-voltage electric fields inside neural membranes and proteins mediate mind-brain input and output processes. In particulars, we suggest that the input processes in said electric fields are possibly mediated by spin transverse forces and/or Dirac-Hestenes electric dipoles both of which are associated with the nuclear/electronic spin processes. We then suggest that the output processes (proactive spin processes) in said electric fields possibly involve Dirac negative energy extraction processes, shown by Solomon, and also Dirac-Hestenes electric dipole interactions of nuclei/electrons besides non-local processes driven by quantum information. We propose that these output processes modulate the action potentials, thus influencing the brain, by affecting the cross-membrane electric voltages and currents directly and/or indirectly through changing the capacitance, conductance and/or battery in the Hudgkin-Huxley model. These propositions are based on our own experimental findings, further theoretical considerations, and studies reported by others in the fields of spintronics, high-energy physics and alternative energy research.
\end{abstract}

Key Words: spin, mind-pixel, electric spin effect, spin transverse force, Dirac-Hestenes electric dipole, electric field, proactive spin

Corresponding author: Huping Hu, Ph.D., J.D.,

Address: Biophysics Consulting Group, 25 Lubber Street, Stony Brook, NY 11790, USA.

E-mail: hupinghu@quantumbrain.org 


\section{Introduction}

Within the framework of spin-mediated consciousness theory, the nuclear/electronic spins are proposed to be the mind-pixels which interact with the brain through quantum effects, modulating and being modulated by various classical brain activities such as the action potentials ( $\mathrm{Hu} \&$ Wu, 2002 \& 2004a-d). Since classical brain activities are largely electric and, in comparison, magnetic fields insides the brain are only microscopically strong but fluctuating, we have previously discussed how action potentials modulate the dynamics of nuclear/electron spin networks inside the brain through $\mathrm{J}$-coupling, dipolar coupling and chemical shielding tensors, thus, feeding information into mind in the dualistic approach (Hu \& Wu, 2004 c \& 2004d). Further, based on our own experimental findings and work done by others, we have also discussed on how mind might influence brain through proactive spin processes enabled by the varying high-voltage electric fields inside the brain (Hu \& Wu, 2006a-d \& 2007a-c).

What we have not explored in details so far are the electric spin effects which are the direct effects of electric fields on the dynamics/motions of nuclear and/or electronic spins and related phenomena, and their possible roles in mind-brain interactions. Thus, we explore here a more specific model of mind-brain interaction within aforesaid framework in which the said electric spin effects in the varying high-voltage electric fields inside neural membranes and proteins mediate mind-brain input and output processes.

In what to follow, we will first briefly describe our experimental results and discuss their implications on mind-brain interaction. We will then suggest that brain-mind input processes in the electric fields are possibly mediated by spin transverse forces and/or Dirac-Hestenes electric dipoles both of which are associated with the nuclei/electron spin processes. We will then suggest that the output processes (proactive spin processes) in said electric fields possibly involve Dirac negative energy extraction processes, as shown by Solomon (2006 \&2007), and also Dirac-Hestenes electric dipole interactions of nuclei and/or electrons besides non-local processes driven by quantum information shown buy us. We suggest that these output processes modulate the action potentials, thus influencing the brain, by affecting the cross-membrane electric voltages and currents directly and/or indirectly through changing the capacitance, conductance and/or battery in the Hudgkin -Huxley model.

\section{Recent Experimental Findings}

In the first set of experiments, we tested the possibility of quantum-entangling the quantum entities inside the brain with those in an external anesthetic sample. We found that applying magnetic pulses to the brain when a general anesthetic sample was placed in between caused the brain to feel the effect of said anesthetic for several hours after the treatment as if the test subject had actually inhaled the same $(\mathrm{Hu} \& \mathrm{Wu}, 2006 \mathrm{~b} \&$ 2006c). We then verified that the said brain effect is indeed the consequence of quantum entanglement between quantum entities inside the brain and those of the chemical substance under study induced by the photons of the magnetic pulses or applied lights (id). We suggest that the said quantum entities inside the brain are nuclear or electronic spins $(i d)$.

In the second sets of experiments, we measured the changes of physical and/or chemical parameters in simple quantumentangled systems. We found that the $\mathrm{pH}$ 
value and temperature of water in a detecting reservoir quantum-entangled with water in a remote reservoir can change against local environment when the latter is manipulated under the condition that the water in the detecting reservoir is able to exchange energy with its local environment $(i d)$. We also found that the gravity of water in a detecting reservoir quantum-entangled with water in a remote reservoir can change against local gravity when the latter was remotely manipulated $(\mathrm{Hu} \& \mathrm{Wu}, 2006 \mathrm{~d} \&$ 2007a).

In the context of mind-brain interaction, our above findings imply that the mind, if it is or behave like a quantum entity as in our spin-mediated consciousness theory, may affect the brain through quantumentanglement mediated non-local processes in defiance of the second law of thermodynamics. Though the details of such interaction still needed to be worked out in future studies, we here give a hypothetical example of how it might be like.

Let us assume that (1) one wants to lift one's finger; (2) the said desire (free will) is represented by quantum information in one's mind; and (3) the mind is quantum-entangled with brain through mind-pixels which are nuclear and/or electron spins inside the brain. Then our experimental results suggest that it is possible for one's mind to affect the action potentials in the brain thus lifting one's finger through nuclear and/or electron spin mediated non-local processes.

\section{Spin Transverse Force}

Recent studies in spintronics have shown that an electric field will exert a transverse torque/force on a moving spin (see, e.g., Sun et al 2004; Shen, 2005). This is actually not hard to understand since according to special theory of relativity a moving spin in an electric field sees a magnetic field.
Sun et al (2004) has shown that a moving spin is affected by an external electric field and feels a force/torque as $\mathbf{m} \times\left[\left(\mathbf{v} / \mathrm{c}^{2}\right) \times \mathbf{E}\right]$ where $\mathbf{m}$ and $\mathbf{v}$ are respectively the magnetic moment and the velocity of the moving spin and $\mathbf{E}$ is the external magnetic field.

Shen (2005) has shown that, as a relativistic quantum mechanical effect, an external electric field exerts a transverse force on an electron spin $1 / 2$ if the electron is moving. The said spin force, analogue to the Lorentz force on an electron charge in a magnetic field, is perpendicular to the electric field and the spin motion when the spin polarization is projected along the electric field (Id).

Indeed, while this paper is been written, this effect has just been successfully used in the laboratory to flip the spin of an electron in a quantum dot by applying an oscillating electric field (Nowack, et al, 2007). The electric field induces coherent transitions (Rabi oscillations) between spin-up and spin-down with $90^{\circ}$ rotations as fast as $\sim 55$ ns and the analysis done by the authors indicates that the electrically-induced spin transitions are mediated by the spin-orbit interaction $(I d)$.

Therefore, we specifically propose here (Proposition I) that the interactions between the moving nuclear/electronic spins in neural membranes and proteins and the varying high-voltage electric fields there directly feed information into mind in the dualistic mind-brain approach of spin mediated consciousness theory.

To illustrate this particular mechanism, we now consider the spin transverse force exerted on a proton spin of a hydrogen atom connected to the carbon chain of a phosphate lipid located inside the neural membranes as shown in Figure 1. As the carbon chain rotates in parallel to the intense electric field 
E across the neural membranes, the vertical proton spin moving in a circle perpendicular to the carbon chain sees a magnetic field in the rotating frame of reference thus feels a transverse torque/force $f$ toward the rotating plane. Quantitative calculations shall be performed in a separate paper.

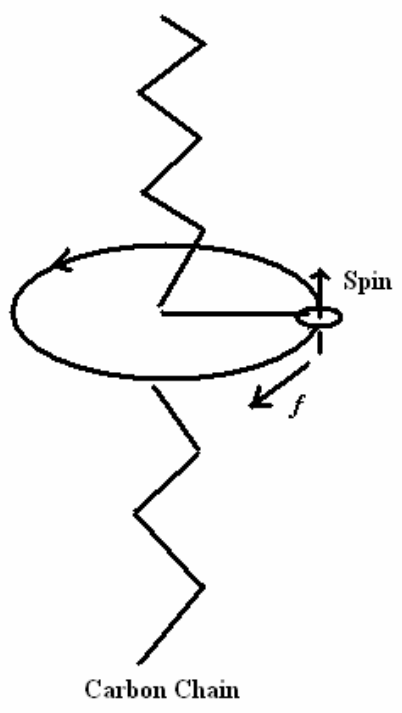

Fig.1. Illustration of spin transverse torque/force $f$ exerted on a nuclear/electronic spin on a molecular chain or fragment inside the neural membranes and proteins.

This spin transverse torque/force enables the neural spike trains to directly influence the nuclear/electronic spin networks in neural membranes and proteins thus inputting information into mind in the dualistic approach.

\section{Dirac-Hestenes Electric Dipole}

It has been long known that in an external electric field, the Dirac particle such as an electron or nuclear sub-entity acts as if it has an imaginary electric moment $|\mathbf{d}|=i \mathrm{e} h / 2 \mathrm{mc}$ where $i$ is the imaginary unit - square root of -1. Dirac was aware this in 1928 and wrote that " $[t]$ he electric moment, being a pure imaginary ... should not...appear in the model [and it] is doubtful whether the electric moment has any physical meaning ..." (Dirac, 1928). Later, Dirac stated that "these extra terms involve some new physical effects, but since they are not real they do not lend themselves very directly to physical interpretation" (Dirac, 1983). We comment here that whether the electric dipole is real or imaginary may depend on the reference frame or representation used.

It was Hestenes who showed that Dirac magnetic and electric dipole moments have same origin associated with spin and magnetization (For a review, see, Hestenes, 2003). In Hestenes' formulism, magnetic moment density is not directly proportional to the spin but "dually proportional." The duality factor $e^{i \beta}$ has the effect of generating an effective electric dipole moment for the Dirac particle. Hestenes commented that "this seems to conflict with experimental evidence that the electron has no detectable electric moment, but the issue is subtle" (Id).

However, while this paper is being revised, Hestenes has just released two papers addressing this very same issue (Hestenes, 2008a \& 2008b). He now proposes a zitterbewegung model which can either be regarded as a quasi-classical approximation that embodies structural features of the Dirac equation or treated as a formulation of fundamental properties of the electron that are manifested in the Dirac equation in some kind of average form. His suggested averaging over zitterbewegung as seen in the rest frame of the Dirac particle contains both a real magnetic dipole and real electric dipole (id). Hestenes further believes that this real electric dipole might already have experimental support (id).

Other researchers have also shown recently that the magnetic and electric dipole moments of a fermion are closely related because they determine the real and 
imaginary part of the same physical quantity (Feng et al, 2001; Graesser \& Thomas, 2002).

Further, Silenko has recently shown in the Foldy-Wouthuysen representation that although the influence of the electric dipole moment on the Dirac particle motion is negligibly small in an external electric field, it influences significantly the spin motion of the said particle (Silenko, 2006).

Furthermore, in the classical models of the Dirac particle, fast oscillating electric dipole moments also appear (Rivas, 2005; Gauthier 2006). These findings coincide with earlier finding that a moving magnetic dipole induces an electric dipole $\mathbf{d}=\left(\mathbf{v} / \mathrm{c}^{2}\right) \times \mathbf{m}$, where $\mathbf{m}$ and $\mathbf{v}$ are respectively the magnetic moment and the velocity of the moving spin, as a relativistic effect (Rosser, 1964). Rivas (2005) believes that what is lacking in the typical quantum mechanical wave equation is this oscillating electric dipole. He states that "in general, the average value of this term in an electric field of smooth variation is zero, [but] in high intensity fields or in intergranular areas in which the effective potentials are low, but their gradients could be very high, this average value should not be negligible." Rivas further showed that the electric moment of the classical Dirac electron could lead to interesting physical effects (Id).

Here we specifically propose (Proposition II) that in the dualistic mindbrain approach of spin-mediated consciousness theory the interactions between the Dirac-Hestenes electric dipoles of nuclei and/or electrons with the varying highvoltage electric fields inside the neural membranes and proteins directly feed information carried by the neural spike trains into mind through the varying high-voltage action potentials.
Do we have any other justifications for Proposition I besides the points already discussed above? The answer, indeed, is "Yes." First, even if the Dirac electric dipole is purely imaginary with no known physical consequence, we argue that in the dualistic mind-brain approach, it may serve as an information receiver in the non-local domain where mind resides for the simple reason that such non-local domain is likely amicable to a description by the imaginary numbers (See, e.g., Rauscher \& Targ, 2001).

Second, we strongly believe that the origin of the electric dipole is intrinsically associated with a Dirac particle actually being a composite entity with the unmanifested negative energy side of the entity inseparably accompanying positive energy side of the entity. We have reason to believe that the un-manifested side of the entity is an active participant in the primordial self-referential spin processes driving quantum mechanics, spacetime dynamics and consciousness as will be discussed elsewhere in due time (Also see, $\mathrm{Hu} \& \mathrm{Wu}$, 2003 \& 2004b).

\section{Output Process through Proactive Spin}

Previously, we have discussed how mind might influence the brain through primordial self-referential spin processes $(\mathrm{Hu} \& \mathrm{Wu}$, 2007c). Our thoughts were that the manifestation of free will is intrinsically associated with the nuclear and/or electron spin processes inside the varying highvoltage environment of the neural membranes and proteins which likely enable the said spin processes to be proactive, that is, being able to utilize non-local energy (potential) and quantum information to influence brain activities through spin chemistry and possibly other chemical and/or physical processes in defiance of the second law of thermodynamics (Id). 
With respect to possible evidence, we pointed out that: (1) our own experiments suggest that non-local energy/potential and quantum information may influence brain function through quantum entanglement mediated processes $(\mathrm{Hu} \& \mathrm{Wu}, 2006 \mathrm{a}-\mathrm{d}$; 2007a); (2) the well-known placebo effect clearly indicates the influence of the mind over body; (3) many experimental reports in parapsychology indicate the plausibility of mind's influences over brain or matter (e.g., Jahn \& Dunn, 2005; Tiller, 2007; Graham, 2006; Radin, 2006); and (4) in the field of alternative energy research, there are numerous papers reporting excess heat being produced through electrophoreses and various plasma discharge schemes the common feature of which is that somehow under the influence of electric fields or high electric voltages, excess heat was claimed to be produced from the vacuum or age-old ether (e.g., Graneau \& Graneau, 1983; Correa \& Correa, 2004).

Here we suggest possible additional mechanisms besides what our own experiments have shown as discussed in Section 2. One such possible additional mechanism is connected to the Dirac negative energy extraction in a varying electric field which has been shown to be theoretically possible by Solomon (e.g., Solomon, 2006 \& 2007).

The vacuum electrons obey the Dirac equation and the energy of these electrons will change in response to an applied electric field (Id.). Solomon has examined the vacuum in Dirac's hole theory and he showed that it is possible to find a varying electric field for which the change in the energy of each vacuum electron is negative (Id.). Therefore, according to Solomon, the total change in the energy of the vacuum state is negative and this new state will have less energy than the original unperturbed vacuum state (id).

Solomon's theoretical results provide support to the claims of excess heat being produced through electrophoreses and various plasma discharge schemes (e.g., Graneau \& Graneau, 1983; Correa \& Correa, 2004). The reviewer of this paper has pointed out that the excess heat in plasma discharges can also be explained in terms of vorticity or torsion fields which had escaped our attention but are closely associated with spin.

We don't think that a vacuum electron in a varying electric field can fall into the negative energy state occupied by the un-manifested side of the composite electron. Thus, we propose (Proposition III) that in certain varying external electric field the nuclear and/or electron spins pump (or create) energy from the vacuum and release the same in our spacetime in order to maintain their minimal energy state in the vacuum. We will discuss where the vacuum energy comes from in a separate paper.

Further, as Rivas (2005) pointed out that what is lacking in the quantum mechanical wave equation is possibly the oscillating electric dipole which in high intensity fields or in intergranular areas should not be negligible in its contributions to dynamics of the Dirac particle. Hestenes (2008a \& 2008b) has just express similar views with his zitterbewegung model of the electron.

The complication is that in the literal interpretation of Dirac equation in an external electric field, the appearance of an imaginary dipole energy term $\mathrm{H}_{\text {int }}=(-i \mathbf{d} \cdot \mathbf{E})$ where $i$ is the imaginary unit in the Hamiltonian makes it non-hermitian and the corresponding energy spectrum complexvalued instead of real valued. However, various studies of the non-hermitian Hamiltotians indicate that not only very interesting novel dynamics appear due to 
non-hermiticity (See, e.g., Aguiar Pinto et al, 2003) but also certain classes of nonhermitian Hamiltonians still have real energy spectra (See, e.g., Bender, 2007). We propose (Proposition IV) that these new dynamics involving Dirac-Hestenes electric dipole may be one of several possible mechanisms needed to enable the proactive spin process.

The reviewer of this paper is well justified to question the legitimacy of imaginary quantities being involved in proactive spin processes which we hope to address in a separate paper. In our defense, we would like to point out that: (1) as discussed above, whether a quantity is real or imaginary in the Hamiltonian depends on the reference frame or representation used; (2) Hestenes' formulism of Dirac equation clearly show that all quantum effects of the electron is intrinsically connected to spin which in turn is tied to imaginary unit $i$. As the reviewer also said, the issue involving imaginary quantities is very difficult. New physics is probably involved as Dirac conjectured but it is worthwhile to keep the option open.

Finally, we propose (Proposition V) that these output processes modulate the action potential, thus influencing the brain, by affecting the cross-membrane electric voltages and currents directly and/or indirectly through changing the capacitance, conductance and/or battery in the Hudgkin-Huxley model.

\section{Conclusion}

Electric spin effects are effects of electric fields on the dynamics of nuclear/electronic spins and related phenomena. In this paper, we have explored several such effects and their possible roles in the mind-brain interactions within the framework of spin mediated consciousness theory. We have outlined five propositions. In particulars, we have considered a more specific model of mind-brain interaction in which these effects in the varying high-voltage electric fields inside neural membranes and proteins mediate mind-brain input and output processes.

We have suggested that the input processes in said electric fields are possibly mediated by spin transverse force/torque and/or Dirac-Hestenes electric dipoles both of which are associated with the nuclear/ electronic spin processes. We then suggest that the output processes (proactive spin processes) in said electric fields possibly involve Dirac negative energy extraction processes, as shown by Solomon, of nuclei/electrons and also Dirac-Hestenes electric dipole interactions in said electric fields besides non-local processes driven by quantum information. We have proposed that these output processes modulate the action potentials, thus influencing the brain, by affecting the cross-membrane electric voltages and currents directly and/or indirectly through changing the capacitance, conductance and/or battery in the HudgkinHuxley model.

These propositions are based on our own experimental findings, further theoreticcal considerations within the framework of spin-mediated consciousness theory, and studies reported by others in the fields of spintronics, high-energy physics and alternative energy research.

Finally, we stress again the importance of experimental work and quantitative calculations and computer simulations in order to verify these propositions and make substantial progresses. We have done some preliminary work in these directions. As usual, results will be reported, when feasible. 
NeuroQuantology 6:1 p26-31 (2008)

$\mathrm{Hu}$ and $\mathrm{Wu}$, Concerning spin as mind-pixel: how mind interacts with the brain through electric spin effects

Acknowledgement: We wish to thank the reviewer for his insightful comments. We

\section{References}

Aguiar Pinto, A C et al. Imaginary phases in two-level model with spontaneous decay. J. Phys. A: Math. Gen.2003; 36: 7461-7467.

Aspden H. Creation: The Physical Truth, Book Guild Publishing (Brighton, England) 2006.

Bender, C M. Making sense of non-hermitian Hamiltonians. Rep. Prog. Phys. 2007; 70: 947-1018.

Correa PN and Correa AN. Experimental Aetheometry v1 (Akronos Pub), 2004.

Dirac, P A M. The quantum theory of the electron. Proc. R. Soc. 1928; A 117: 610-624.

Dirac, P A M. The Principles of Quantum Mechanics (4th edition) 1983; (Clarendon Press, Oxford, p. 265)

Feng, el al. Theoretical expectations for the muon's electric dipole moment.Nucl.Phys.2001; B613: 366-381.

Gauthier R. The Dirac equation and the superluminal electron model. 2006; http://superluminalquantum.org

Graesser, M. \& Thomas, S. Supersymmetric relations among electromagnetic dipole operators. Phys. Rev. 2002; D65: 075012.

Graham D. Experimental data demonstrating augmentation of ambient gravitational and geomagnetic fields. STAIF 2006.

Graneau P \& Graneau PN. First indication of Ampere tension in solid electric conductors. Phys Lett 1983; 97A: 253.

Hestenes D. Spacetime physics with geometric algebra. Am. J. Phys. 2003; 71 (6): 691-714. have revised this paper accordingly to expand or clarify certain concepts.

Hestenes D. Zitterbewegung in Quantum Mechanics -a research program. arXiv 2008a; [quant-ph] 0802.2728 .

Hestenes D. Reading the Electron Clock. arXiv 2008a; [quant-ph] 0802.3227.

$\mathrm{Hu} \mathrm{H}$ and $\mathrm{Wu} \mathrm{M}$. Spin-mediated consciousness theory. arXiv 2002; quant-ph/0208068. Also see Med. Hypotheses 2004a: 63: 633-646.

$\mathrm{Hu} \mathrm{H}$ and $\mathrm{Wu} \mathrm{M}$. Spin as primordial self-referential process driving quantum mechanics, spacetime dynamics and consciousness. NeuroQuantology 2004b; 2:41-49. Also see Cogprints: ID2827 2003.

$\mathrm{Hu} \mathrm{H}$ and $\mathrm{Wu}, \mathrm{M}$. Action potential modulation of neural spin networks suggests possible role of spin in memory and consciousness. NeuroQuantology 2004c; 2:309-316. Also see Cogprints: ID3458 2004d.

$\mathrm{Hu} \mathrm{H}$ and $\mathrm{Wu}, \mathrm{M}$. Thinking outside the box: the essence and implications of quantum entanglement. NeuroQuantology 2006a; 4: 5-16.

$\mathrm{Hu} \mathrm{H}$ and $\mathrm{Wu} \mathrm{M}$. Photon induced non-local effect of general anesthetics on the brain. NeuroQuantology 2006b; 4:17-31. Also see Progress in Physics 2006c; v3: 20-26.

$\mathrm{Hu} \mathrm{H}$ and $\mathrm{Wu} \mathrm{M}$. Evidence of non-local physical, chemical and biological effects support quantum brain. NeuroQuantology 2006d; 4: 291-306. Also see Progress in Physics 2007a; v2: 17-24.

$\mathrm{Hu} \mathrm{H} \& \mathrm{Wu}$ M. Thinking outside the box II: the origin, implications and applications of gravity and its role in consciousness. NeuroQuantology 2007b; 5:190-196.

$\mathrm{Hu} \mathrm{H} \& \mathrm{Wu} \mathrm{M}$. How mind influences brain through proactive spin. NeuroQuantology 2007c; 5: 205-213.

Jahn R G and Dunne B J. The PEAR proposition. J Sci Exploration 2005;19: 195-245. 
NeuroQuantology 6:1 p26-31 (2008)

$\mathrm{Hu}$ and $\mathrm{Wu}$, Concerning spin as mind-pixel: how mind interacts with the brain through electric spin effects

Kozyrev N A. Sources of Stellar Energy and the Theory of the Internal Constitution of Stars. Progress in Physics 2005; v3: 61-99.

Nowack, et al. Coherent control of a single electron spin with electric fields. Science Express 2007; DOI: 10.1126/science. 1148092.

Radin D. Entangled mind: extrasensory experiences in a quantum reality. Paraview Pocket Books 2006.

Rauscher, E A \& Targ, R. The speed of thought: Investigation of a complex space-time metric to describe psychic phenomena. J. Sci. Explor. 2001; 15: 331-354.

Rivas M. Kinematical formalism of elementary spinning particles. arXiv 2005; physics/0509131.

Rosser, W G V. An introduction to the theory of relativity, P326. London Butterworths, Press 1964.

Shen, S Q. Spin transverse force on spin current in an electric field. Phys. Rev. Lett. 2005; 95: 187203.

Silenko, A J. Quantum-mechanical description of the electromagnetic interaction of relativistic particles with electric and magnetic dipole moments. Russ.Phys.J. 2005; 48: 788-792.

Solomon, D. Some new results concerning the vacuum in Dirac Hole Theory. arXiv 2006; quant-ph/0607037.

Solomon, D. Quantum states with less energy than the vacuum in Dirac Hole Theory. arXiv 2007; quant-ph/0702271.

Sun, Q F et al. Spin-current-induced electric field. Phys. Rev. B 2004; 69: 054409.

Tiller W. Psychoenergetic science: a second Copernican-scale revolution. Accessed 2007, May. http://www.tiller.org 